\title{
NLOS GPS Signal Detection Using A Dual-Polarisation Antenna ${ }^{1}$
}

\author{
Ziyi Jiang, Paul D Groves \\ University College London, United Kingdom \\ Email: ziyi.jiang@ucl.ac.uk \\ Tel: $+44(0) 2076793638$
}

\begin{abstract}
The reception of indirect signals, either in the form of non-line-of-sight (NLOS) reception or multipath interference, is a major cause of GNSS position errors in urban environments. We explore the potential of using dual-polarisation antenna technology for detecting and mitigating the reception of NLOS signals and severe multipath interference. The new technique computes the value of the carrier-power-to-noise-density $\left(C / N_{0}\right)$ measurements from left-hand circular polarised (LHCP) outputs subtracted from the right-hand circular polarised (RHCP) $C / N_{0}$ counterpart. If this quality is negative, NLOS signal reception is assumed. If the $C / N_{0}$ difference is positive, but falls below a threshold based on its lower bound in an open-sky environment, severe multipath interference is assumed. Results from two experiments are presented. Open field testing was first performed to characterise the antenna behaviour and determine a suitable multipath detection threshold. The techniques were then tested in a dense urban area. Using the new method, two signals in the urban data were identified as NLOS-only reception during the occupation period at one station, while the majority of the remaining signals present were subject to a mixture of NLOS reception and severe multipath interference. The point positioning results were dramatically improved by excluding the detected NLOS measurements. The new technique is suited to a wide range of static ground applications based on our results.
\end{abstract}

\section{Keywords}

GPS, Non-Line-Of-Sight, NLOS, Multipath, Dual-polarisation Antenna

\section{Introduction}

The urban environment presents two major challenges to GNSS signal reception. Firstly, buildings and other obstacles, such as buses, block the direct line-of-sight (LOS) to many satellites, reducing the number in view. The remaining signals often have poor geometry, degrading the positioning accuracy, while a multi-constellation receiver is essential in order to reliably obtain sufficient direct-LOS signals to compute a position solution (Wang 2012).

The second problem is that urban environments contain many flat surfaces that reflect GNSS signals. Tinted glassy buildings are particularly strong reflectors. Reception of these

\footnotetext{
${ }^{1}$ This is the author archive version of the same paper published on GPS Solutions (DOI:

10.1007/s10291-012-0305-5). The final publication is available at http://link.springer.com.
} 
reflected signals results in significant positioning errors. Where the direct signal is blocked and the signal is received only via reflections, this is known as non-line-of-sight (NLOS) reception. Where the signal is received through multiple paths, this is known as multipath interference. NLOS reception and multipath interference are often grouped together as "multipath". However, they are actually separate phenomena that produce very different ranging errors. NLOS reception and multipath interference can occur separately, but also occur together whenever a signal is received via multiple reflected paths but not directly.

NLOS reception results in a pseudo-range measurement error equal to the additional path delay, the difference between length of the path taken by the reflected signal and the (blocked) direct path between satellite and receiver. This error is always positive and, although typically tens of metres, is potentially unlimited. Signals received via distant tall buildings can exhibit errors of more than a kilometre. The corresponding carrier-based ranging error is within half a wavelength of the pseudo-range error (noting that phase changes occur on reflection). NLOS signals can be nearly as strong as the directly received signals, but can also be very weak. As high-sensitivity receivers can acquire much weaker signals, their use can significantly increase the number of NLOS signals received.

Where multipath interference to directly received signals occurs, the reflected signals distort the code correlation peak within the receiver such that the code phase of the direct LOS signal cannot be accurately determined by equalising the power in the early and late correlation channels. The resulting code tracking error depends on the receiver design as well as the direct and reflected signal strengths, path delay and phase difference, and can be up to half a code chip (Groves 2008, Braasch 1996 and Van Nee 1992). Carrier-phase tracking errors are limited to half of a wavelength (assuming the direct LOS signal is stronger than the reflections and a Costas discriminator).

Several methods exist for mitigating multipath interference and NLOS reception. However, they all have their limitations. Techniques may be classified as antenna-based, receiver-based and post-receiver, and may be used in combination. Antenna-based techniques such as beam-forming antenna arrays (Brown 2001), ground planes and choke rings are usually bulky and expensive. Receiver-based techniques, summarised in Groves (2008), that sharpen the peak of the code correlation function are relatively expensive to implement and have no effect on NLOS signal reception.

Post-receiver techniques operate using the pseudo-range, carrier-phase and carrier-powerto-noise density ratio, $C / N_{0}$, measurements prior to the positioning calculation. Multipath interference may be detected and mitigated by comparing measurements on different frequencies from the same satellite (Lau 2007). However, these techniques are of little use for mitigating NLOS reception.

Detection of NLOS reception using a standard receiver requires signals from different satellites to be compared. Consistency-checking techniques (Jiang 2011) can identify and eliminate NLOS signals when most of the other received signals are direct LOS with minimal multipath interference, but are less reliable in the more challenging environments. $C / N_{0}$ can also be used as an indicator of NLOS signals. However, direct-LOS signals may also be attenuated by trees, body masking and low antenna gain in certain directions. Furthermore, diffracted signals, which are normally weaker, are common in urban areas and can be useful for non-precision applications (Wang 2012). Further research on NLOS detection is therefore needed to achieve reliable, cost-effective and accurate urban positioning. 
We explore the potential of dual-polarisation antenna technology for detecting and mitigating both NLOS reception and severe multipath interference. Firstly, we present the background for this study including a review of previous work using dual-polarisation antennas. Secondly we summarise the principle ideas of this research, and describe the use of a dual-polarisation antenna with $C / N_{0}$-based discriminators. Then we describe the process of calibrating the $C / N_{0}$-based discriminators used in this research. The results of a NLOS and multipath detection experiment conducted in a dense urban area are shown in the results section using the same antenna calibration. A brief summary of the work and the plans for further research are presented in the last section.

\section{Background}

GNSS signals are circularly polarised to mitigate the effect of Faraday rotation caused by the earth ionosphere and magnetic field (Hofmann-Wellenhof 2008). Direct signals received from the satellites are right-hand circular polarised (RHCP). On reflection from a building, signals with incidence angles less than Brewster's Angle become LHCP, with the rest remains RHCP (Brenneman 2011). Except for cell phone antennas, which are usually linearly polarised, conventional GNSS antennas are designed to have a higher gain for RHCP signals, attenuating the reception of LHCP signals. This reduces the impact of multipath interference to direct-LOS signals. Conversely, an antenna with the opposite polarisation (LHCP) sensitivity will generally attenuate the direct signals more than the reflected signals.

Dual-polarisation GNSS antennas combine coaxial RHCP-sensitive and LHCP-sensitive antennas in a single housing with two outputs: a conventional output that is more sensitive to RHCP signals and an additional output that is more sensitive to LHCP signals. The assumption is that the overall received signal (reflected plus direct signal) will have a combination of both LHCP and RHCP components. Heavily contaminated signals will have stronger LHCP components and hence have a stronger output on the LHCP antenna feed. By processing the two outputs separately in a GNSS receiver and comparing the measurements obtained, clean direct-LOS, multipath-contaminated direct-LOS and NLOS signals may be distinguished.

A few previous studies have investigated the use of a dual-polarisation antenna to detect and mitigate multipath errors. However, a successful demonstration of NLOS error mitigation using the $C / N_{0}$ difference has yet to be reported.

The use of dual RHCP and LHCP GPS antennas for studying multipath was first proposed in Manandhar (2004) and results were presented using a pair of lab-manufactured helical antennas with commercial receivers. A stronger response for low-elevation satellites and reflected signals from the LHCP antenna compared to the RHCP antenna was demonstrated.

In Izadpanah (2008), an Antcom dual-polarisation antenna was used for real data collection. It was validated, using another multipath detection method, that the LHCP component of the dual-polarisation antenna receives a greater reflected signal power than the RHCP component.

Multipath mitigation using a dual-polarisation antenna in the correlator domain was demonstrated by simulation in Yang (2005). A dual polarisation antenna, a dual-channel coherent RF front-end and a master-slave baseband signal processor were simulated. No more than two multipath components were considered in the simulated signal. 
In Brenneman (2011), multipath mitigation using a LHCP antenna array was assessed by simulation only. The simulated signal included only one multipath component. The estimation of the multipath angle of arrival was demonstrated, and used for multipath mitigation in conjunction with a null-steering algorithm and known angle of arrival of the direct signal.

While previous studies on using dual-polarisation antennas presented successful simulation results, real-world NLOS and multipath reception in urban environment is typically more complicated and chaotic than simulations where only limited geometrical raytracing was considered.

The standard signal-to-noise measure used within the GNSS community is the carrierpower-to-noise-density ratio, $C / N_{0}$. This is defined as the ratio of the received signal power to the weighted noise power spectral density (Groves 2008 and Kaplan 2008). In this research, we exploit the differences in measured $C / N_{0}$ of the same signal from different polarisation outputs of the antenna to detect NLOS reception and multipath interference.

Due to differences in measurement methods, two different types of receiver connected to the same antenna and tracking the same signal at the same time may report different $C / N_{0}$ values. Therefore, when using the dual-output dual-polarisation antenna for multipath and NLOS detection purposes, the same user equipment design for both output feeds.

\section{Dual-Polarisation Antenna For NLOS and Multipath Detection and Mitigation}

An initial experiment described in Groves (2010) showed that $C / N_{0}$ measurements obtained by separately correlating the RHCP and LHCP outputs of a dual-polarisation GNSS antenna can be used to distinguish between a low-multipath/low-NLOS and moderatemultipath/moderate-NLOS environment. In this work, an improved $C / N_{0}$-based detection scheme is used to identify the NLOS signals in a severe-multipath/severe-NLOS environment, which are then excluded from the position solution, improving the accuracy significantly.

The first step is to characterise the performance of the system in a low-multipath/lowNLOS environment. A pair of receivers of the same type is attached to both feeds of the dualpolarisation antenna as shown in Fig.1. The $C / N_{0}$ values are measured and their variation with the elevation angle assessed. A curve is fit through the measured RHCP-LHCP $C / N_{0}$ difference; the lower bound of the $95 \%$ confidence level of the fit can be used as a threshold for later comparisons (as shown below). 


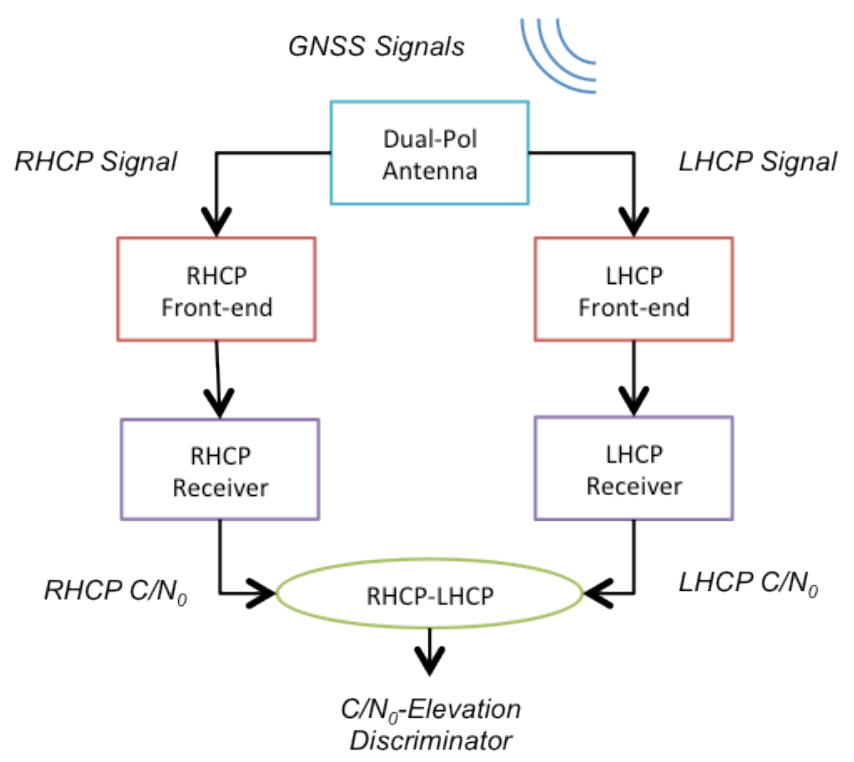

Fig.1 A schematic set-up of the experiment

Depending on the specific antenna type, the gain pattern may vary as a function of both signal elevation and azimuth. It may be calibrated in an Anechoic chamber.

For data collected in a severe-multipath/severe-NLOS environment using the same set-up, the measured $C / N_{0}$ difference may be compared with the low-multipath/low-NLOS $C / N_{0^{-}}$ based statistics at the corresponding elevation angle to obtain an estimate of the level of interference caused by the reception of reflected signals. Three cases are considered, as illustrated in Fig.2:

1) Similar Multipath: If the measured $C / N_{0}$ difference lies within the $95 \%$ bounds of the fitted function, then it may be assumed that the signal is likely to be subject to a low multipath interference level similar to that in the calibration environment. The $C / N_{0}$ difference can sometimes lie above the upper bounds due to azimuth variation on the antenna gain. This is also classified as low-multipath interference.

2) Severe Multipath: If the $C / N_{0}$ difference is positive, but lies below the lower bound of the fitted function, then there is a significant probability that the signal is subject to a more severe multipath environment than the calibration environment.

3) NLOS Reception: If the $C / N_{0}$ difference is negative, then it is likely that the direct LOS signal is blocked and only reflected signals are being received from that satellite.

Therefore, by comparing the measured RHCP-LHCP $C / N_{0}$ difference at two different environments using two thresholds, i.e. lower $95 \%$ bound of the fitted function and the line of zero difference; the current signal reception category may be determined. 


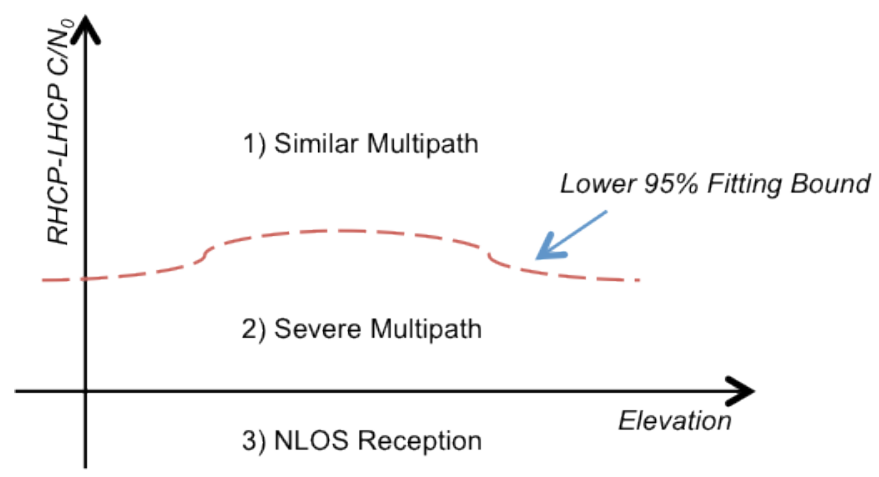

Fig.2 Three cases of the RHCP-LHCP $C / N_{0}$ comparison

When a NLOS signal is identified through the scheme presented in Fig.2, the most straightforward action is to remove it from the measurement set for the final solution because the scale of the error due to NLOS reception is usually difficult to measure without further aiding information. Additional techniques, such as consistency checking (Jiang 2011), can also be used to cross check the result.

For a signal detected with severe multipath contamination, different approaches could be applied depending on the number of available signals. When a large number of GNSS signals are available, a contaminated signal could be simply excluded in the same manner as the NLOS signals. For situations where only limited signals are available, techniques using the receiver tracking loop outputs from the two polarisations to estimate the multipath parameters could be applied to correct the multipath error.

\section{System Calibration}

The first step of the NLOS and multipath detection process is to establish a $C / N_{0}$-based discriminator using the experimental set-up as shown in Fig.1 in a low-multipath environment. Two thresholds as described in the Background section are derived from the RHCP-LHCP $C / N_{0}$ difference. This will later be used to compare against data collected in an urban area using the same set-up.

\section{Experiment Set-up}

The antennas used in the experiment were a pair of Antcom dual-polarisation L1/L2 GPS antennas. One of them was attached to a standard tribrach mount with dual amplifiers, as shown in the left panel of Fig.3. The right panel of Fig.3 shows the gain pattern of the antenna at L1 frequency, as provided by Antcom. A pair of Leica System SR530 geodetic GPS receivers, shown in Fig.4, was used for recording data. One receiver was connected to each polarisation output of the antenna via an amplifier powered from the receiver. 

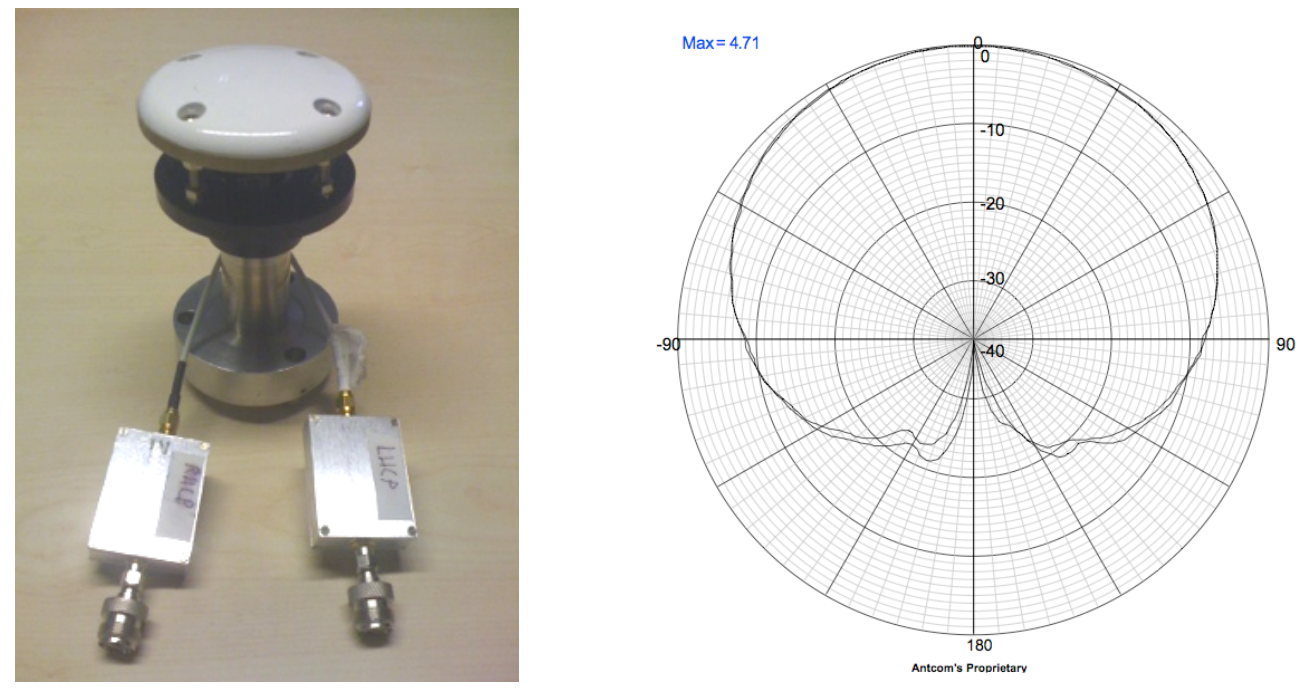

Fig.3 Antcom dual-polarisation antenna with mounting and amplifiers and its gain pattern at L1 frequency

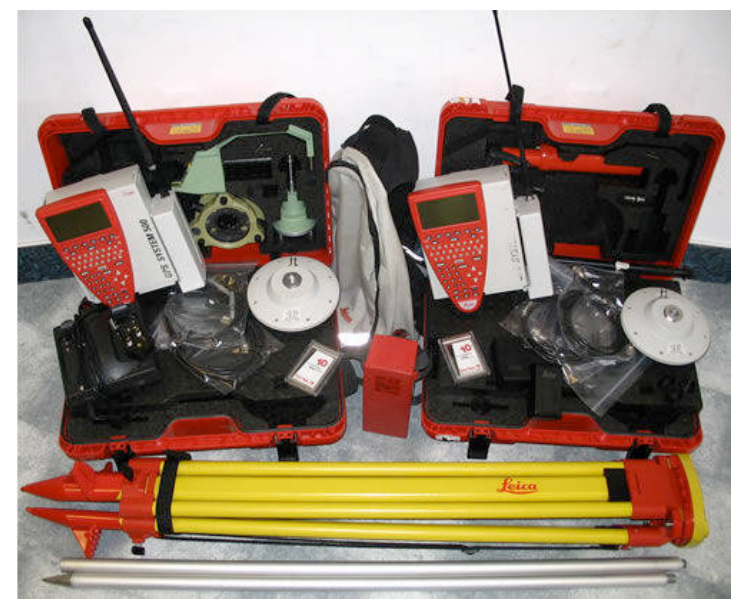

Fig.4 Dual Leica System SR530 geodetic GPS receivers

For some of the tests, an Ashtech choke ring antenna was also used, in order to improve the LHCP signal reception and produce a better quality discriminator. The other Antcom antenna was used with same tribrach, amplifiers and GPS receivers.

\section{Tests In A Low Multipath/NLOS Environment}

A location within London's Regent's Park was selected as a minimal multipath environment for testing and calibrating the dual-polarisation user equipment, as indicated by the yellow marker in Fig.5. Although this location was far away from buildings, there were trees nearby that could affect reception of low-elevation signals. The data used was collected on two consecutive days within the same satellite ground track period. The length of point occupation was 5 hours on both days. The choke-ring-mounted Antcom antenna was used for both days.

The first day of data collection was static data collection with the antenna orientation fixed, i.e. pointed to the magnetic North. To test the hypothesis of the antenna azimuth gain variation, an experiment was conducted on the second day of the data collection in Regent's 
Park. A marking was drawn at the same position on the antenna as an orientation reference. During the data collection, the orientation of the antenna was rotated clockwise by $45^{\circ}$ every 30 minutes from the magnetic North. The angle of rotation and direction was measured with a magnetic compass.

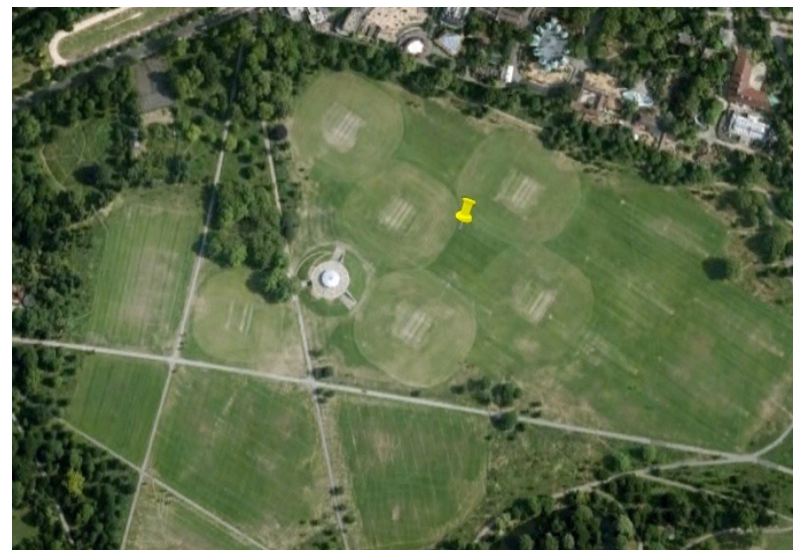

Fig.5 The data collection point at Regent's Park (January 18-19, 2011)

Antenna Azimuth Gain Variation

An example of the results is shown in Fig.6. Both graphs present the $C / N_{0}$ values of the same satellite from both polarisations and their difference plotted against time. The top graph shows the data from the first day of collection with the antenna oriented towards North. The bottom graph shows the data from the second day with antenna orientation changed regularly. The epochs at which the orientation was changed are marked on the right graph as solid vertical lines with the new orientation indicated next to the line.

As can be see from Fig.6, the variation in gain with azimuth occurs mainly on the LHCP feed and the scale of the variation can be as large as $10 \mathrm{~dB}-\mathrm{Hz}$ depending on the orientation. Therefore an optimal $C / N_{0}$ polarisation threshold for this antenna type needs to be modelled as a function of azimuth as well as elevation. This would require an extensive calibration process and constant measurement of the antenna azimuth with respect to North while in use. For these experiments, the antenna was always aligned with North using the alignment mark and a magnetic compass. The need for of an azimuth dependent calibration was circumvented by exploiting the sidereal reception of the GPS satellite azimuth and using single-satellite thresholds. 

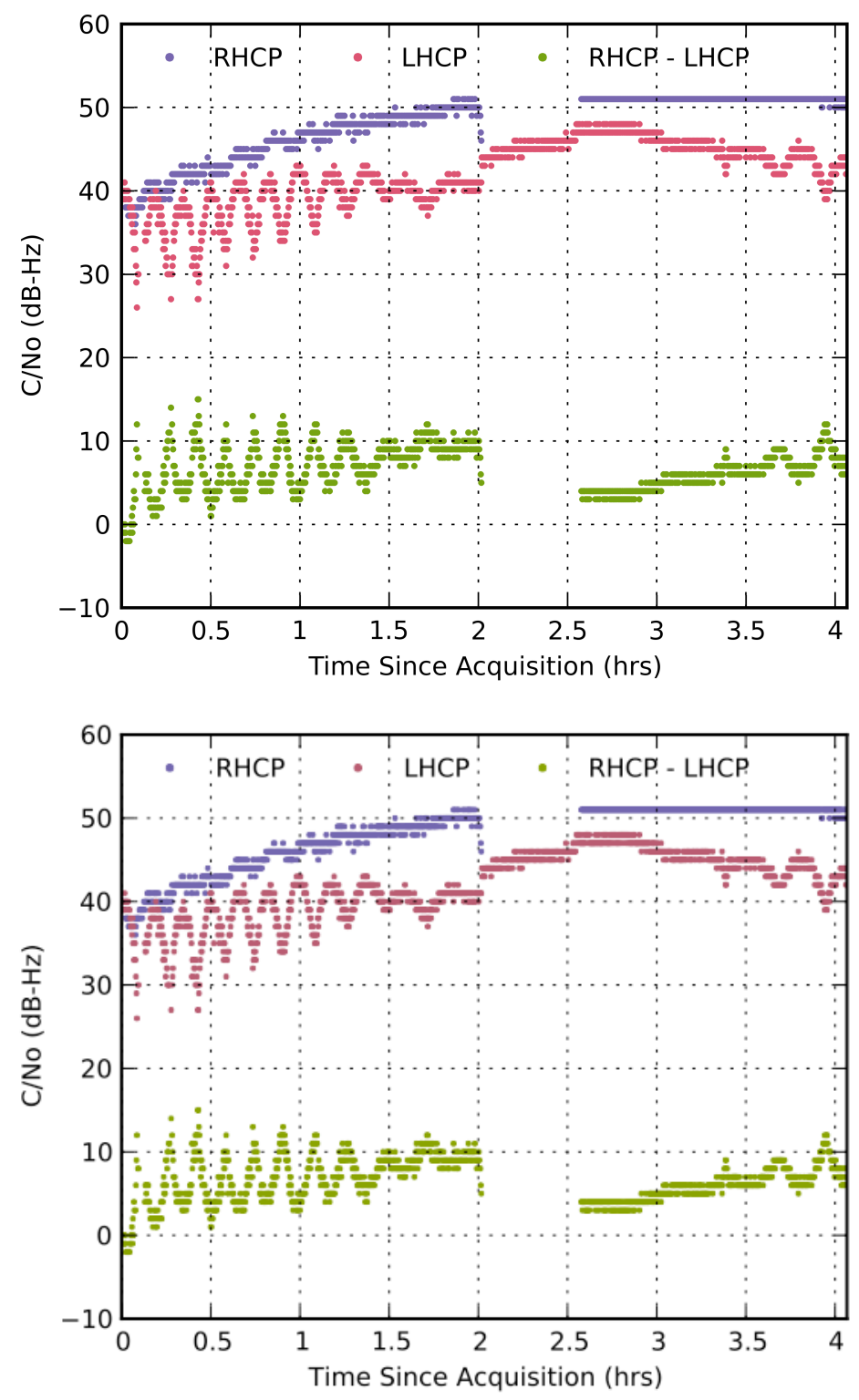

Fig.6 A comparison of the antenna gain variation with respect to the signal azimuth

\section{Individual Satellite Thresholds}

The complete set of data from Regent's Park, expressed against the signal elevation angle, is shown in Fig.7. $C / N_{0}$ values from both RHCP and LHCP feeds and their differences for all tracked satellites are plotted in the graph. As can be seen, there is very little variation in the $C / N_{0}$ difference with lower elevation using the choke ring. However, large variations in $C / N_{0}$ difference can still be observed mainly due the LHCP feed. The main reason for this can be attributed to the antenna gain variation with respect to the signal azimuth. This gain variation has been shown in the antenna calibration stage. Because the overall data collection period lasted for 5 hours, some satellites were tracked during their entire period in view from the user antenna. Because of the difference in the azimuth as the satellite rises and sets, different levels of the $C / N_{0}$ value were often recorded from the same satellite. Differences in the $C / N_{0}$ level were also observed from different satellites with the same elevation, but in different azimuths. 


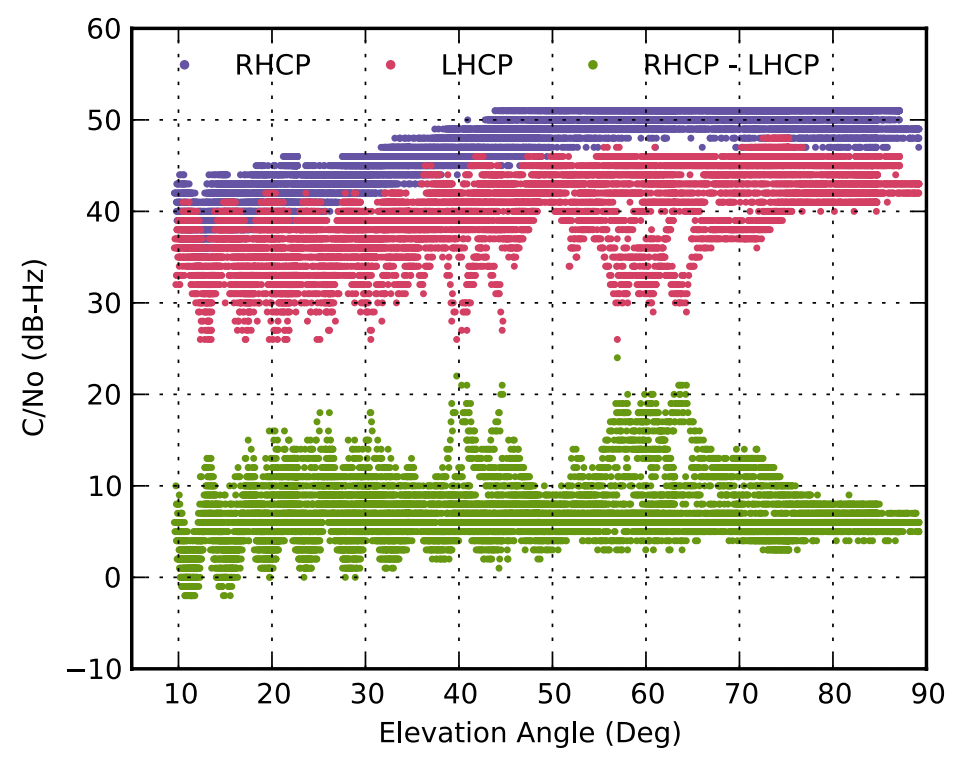

Fig.7 The $C / N_{0}$ data collected in Regent's Park

To improve the sensitivity of NLOS and multipath detection, $C / N_{0}$-based threshold based on individual satellite instead of all satellites in view were derived. Because of the azimuth gain variation, the difference between the upper and lower 95\% fitting confidence boundaries is too large to be useful for multipath detection purposes.

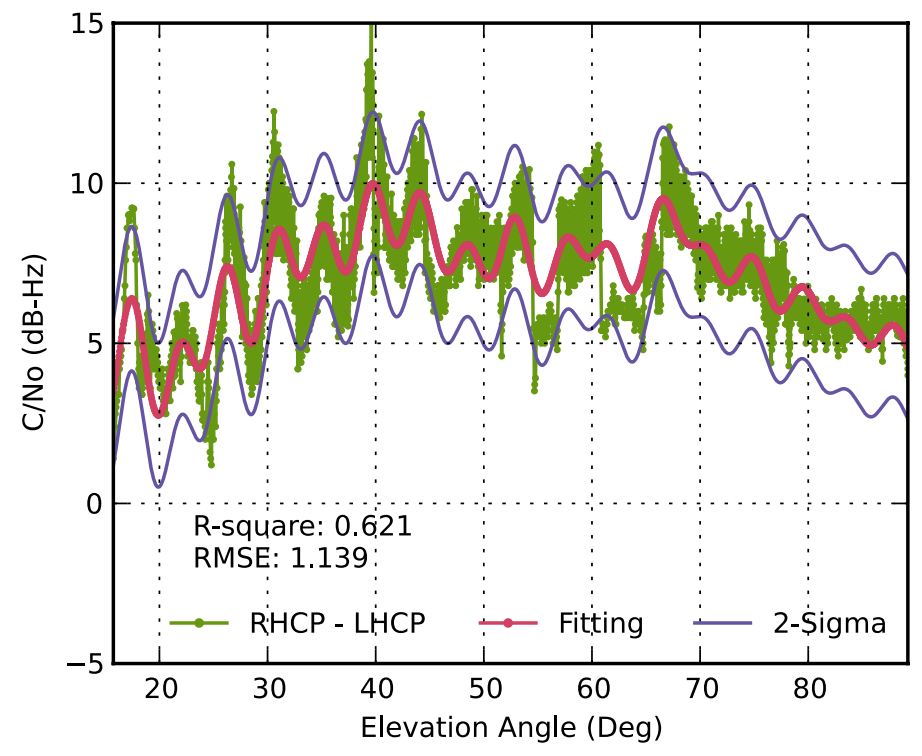

Fig.8 The $C / N_{0}$-based discriminator for $\mathrm{G} 20$

The $C / N_{0}$ data was ats first cleaned for outliers and smoothed. The MATLAB Curve Fitting Toolbox was used to estimate the mean RHCP-LHCP polarisation discrimination as a function of elevation angle from the measured $C / N_{0}$ difference. The $95 \%$ confidence level boundaries were determined at the same time. The fitting function used in this case was an $8^{\text {th }}$ order sine Fourier series. The reason for choosing this function is because it provides the smallest overall fitting errors amongst all the available options of the toolbox. An example of the derived discriminator for the PRN 20 satellite is shown in Fig.8. The lower 95\% boundary was used as a discriminator threshold for later experiments. 


\section{NLOS and Multipath Detection Tests}

The second stage of this study was to compare the derived $C / N_{0}$-based signal discriminators with data collected in an urban environment in order to test the detection capability of the dual-polarisation method.

\section{Experiment Set-up}

The data collection locations were located round Finsbury Square close to Moorgate Tube Station in the City area of London as shown in Fig.9. Prior to the data collection using dualpolarisation receivers, a few truth points were first established on the site using total stations and traversing techniques. These surveyed points are marked as pins in Fig.9 on the traversing loop. Yellow pins in the figure are reference points in an open-space square established using RTK GNSS processing and Ordnance Survey reference station measurements. Blue pins are traverse points that were used to establish the position of the data collection points. Green pins are sites used for data collection. The accuracy of the truth point coordinates is determined by the quality of the surveying ( 1 in 56000) and is at centimetre level.

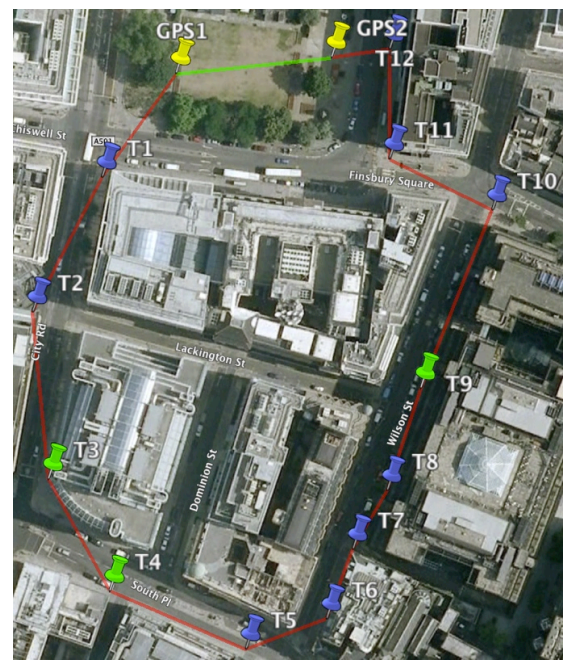

Fig.9 The Finsbury Square data collection site

The data collection using a dual-polarisation antenna was carried out a few weeks after the Regent's Park data. The same hardware set-up as described in previous section was used except without the choke ring in order to maximise the sensitivity of the LHCP feed to NLOS and multipath reception. The data collection times were chosen in order to match the satellite ground track to the data collected in Regent's Park.

Three points were chosen to occupy for data collection including two points (T4 and T9) with different street orientations and a point (T3) in a crossroad right next to a high glassy building. All three points are located in typical urban canyon environments. The surrounding environments of all three occupied points are shown in Fig.10. 

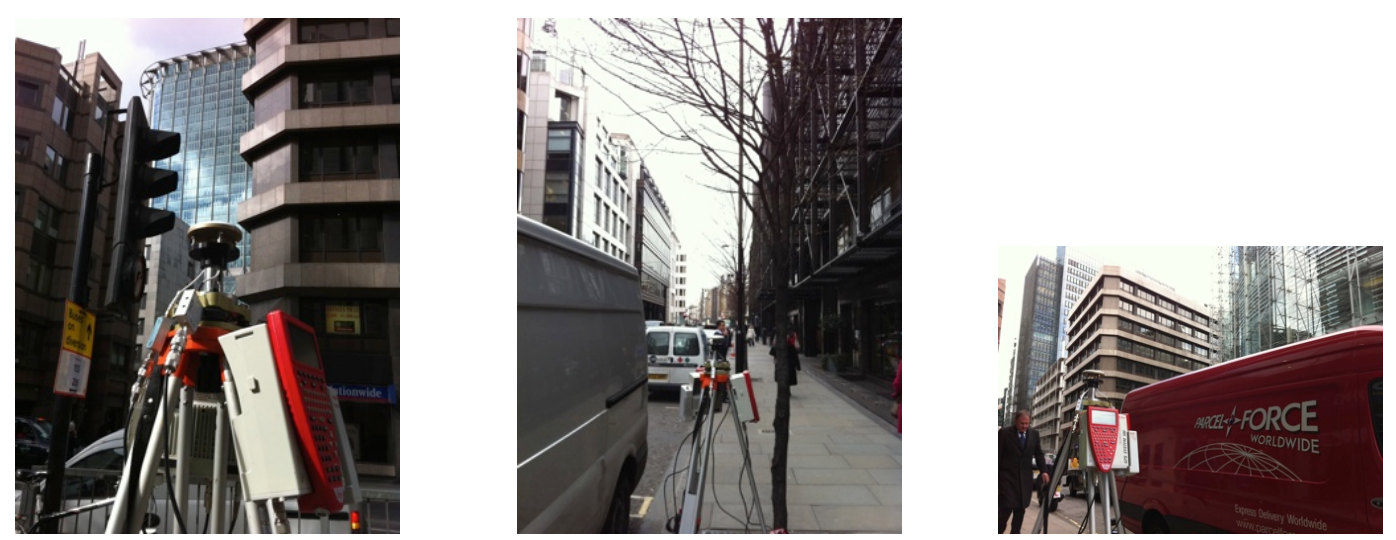

Fig. 10 The three occupied points: left (T3), right (T4), centre (T9)

\section{Test Results}

A few things were immediately noticeable from the urban data. The first is the reduced number of tracked satellites compared to an open environment. This is expected, as chosen points were in typical urban canyons where the buildings block many of the line-of-sights. The average number of GPS satellites tracked at each station was 6 to 7 . The second point is that the different polarisation feeds occasionally lost track of signals at different times, so the amount of time that both the RHCP and LHCP feeds provided $C / N_{0}$ measurements was limited.

Despite these limitations, many successful comparisons were made between the LHCP and RHCP data. Both NLOS reception and multipath contamination were verified using the derived threshold as described in the System Calibration and Tests sections.

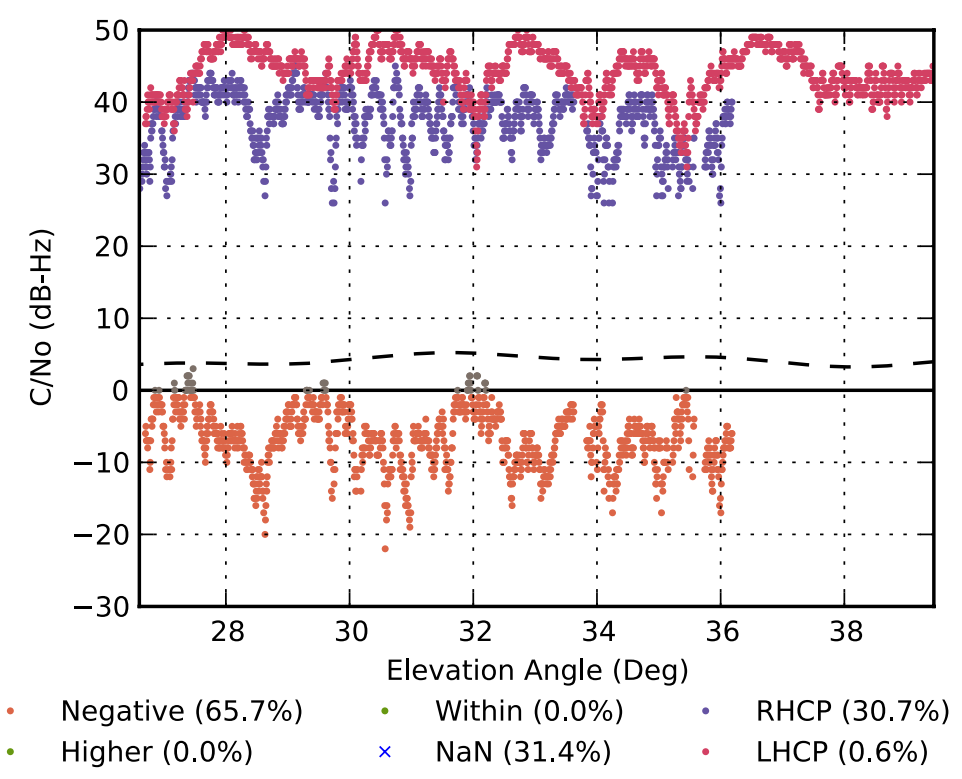

Fig.11 One identified NLOS reception (G24) at T3

Fig.11 shows an example of a NLOS-reception signal identified using the $C / N_{0}$-based thresholds. This was the L1 C/A code signal from PRN 24 received at point T3. The $C / N_{0}$ measurements from the RCHP and LHCP feeds are plotted on the top part of the graph, and the threshold based on the $95 \%$ lower bound in the open environment is drawn as a dashed 
line. The measured RHCP-LHCP $C / N_{0}$ difference is plotted alongside to show the comparison.

A RCHP-LHCP $C / N_{0}$ difference which is less than the lower $95 \%$ bond threshold but greater than zero is classified as severe multipath reception and is shown in grey dots. A negative $C / N_{0}$ difference, shown in orange x marker, is classified as NLOS reception.

As shown in the figure, the identified signal is from a low-elevation satellite. In the majority of epochs $(65.7 \%)$, the $C / N_{0}$ difference is negative, and is therefore identified as NLOS reception based on the criteria illustrated in Fig.2. It can also be observed that the LHCP feed in this case consistently has a better tracking performance than the RHCP feed. The LHCP feed only lost track of the signal for $0.6 \%$ of the overall epochs in comparison with $30.7 \%$ of lost tracking on the RHCP feed. Maintenance of tracking lock on the LHCP feed but not the RHCP feed can also be interpreted as an indicator of NLOS reception.

The NLOS reception identified as shown in Fig.11 can be verified by examine the physical environment surrounding the receiver location at point T3. The left and centre panel of Fig.12 show the environment surrounding the point T3. North and satellite azimuth area during the occupation period are marked by red lines and the direct line-of-sight is marked by a blue line in the centre panel of Fig.12. The right panel of Fig.12 shows a skyplot of the satellite track.

As demonstrated in left panel of Fig.12, the T3 point is located at a junction next to a reflective tall building where reception from most of the southern half of the sky was blocked (Fig.12, centre panel). The satellite ground track of PRN 24 suggests that the satellite should be in the southeast part of the sky from the T3 point rising toward the northwest side of the sky. The azimuth of the satellite varies from $128^{\circ}$ to $135^{\circ}$ (as marked out by red lines in Fig. 12), and elevation is between $25^{\circ}$ and $36^{\circ}$. The building marked by yellow arrows in the figure would block the LOS reception.
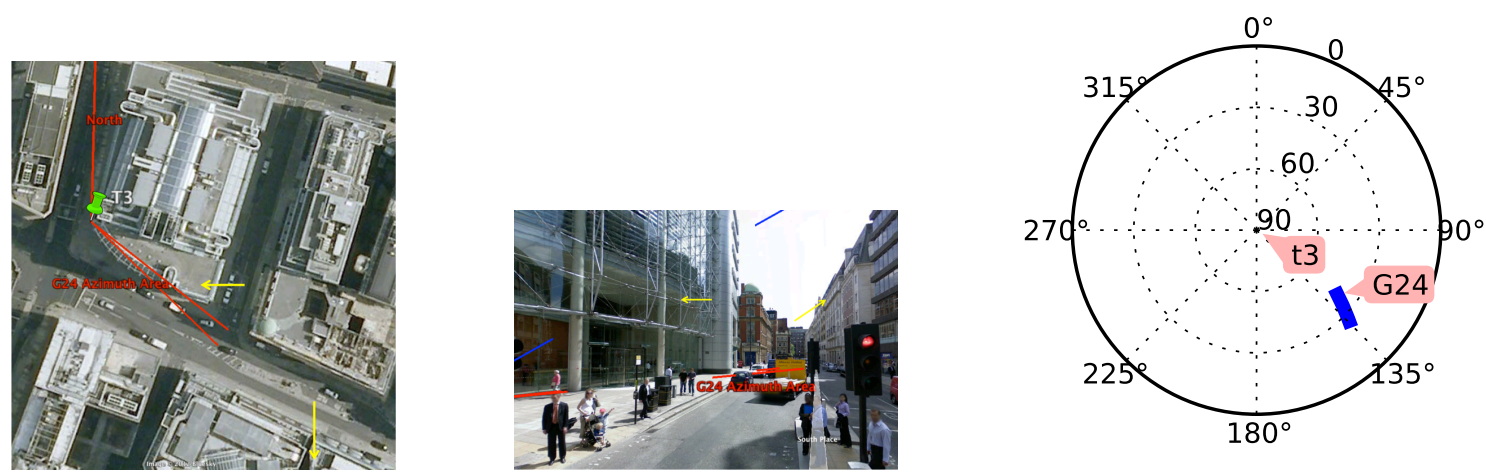

Fig.12 T3 site surroundings and G24 ground track

Fig. 13 shows an example of a signal identified to be contaminated by severe multipath interference compared to Regent's Park where the discriminator was derived. The identified signal was from PRN 20 and received at point T4. Following the same convention as in Fig.11, the original $C / N_{0}$ values from both feeds are respectively plotted on the top part of the graph, and the threshold is drawn as a dashed line. The $C / N_{0}$ difference is plotted alongside the discriminator in colours according to the deduced signal reception category. Green dots indicate no multipath or weak multipath. 


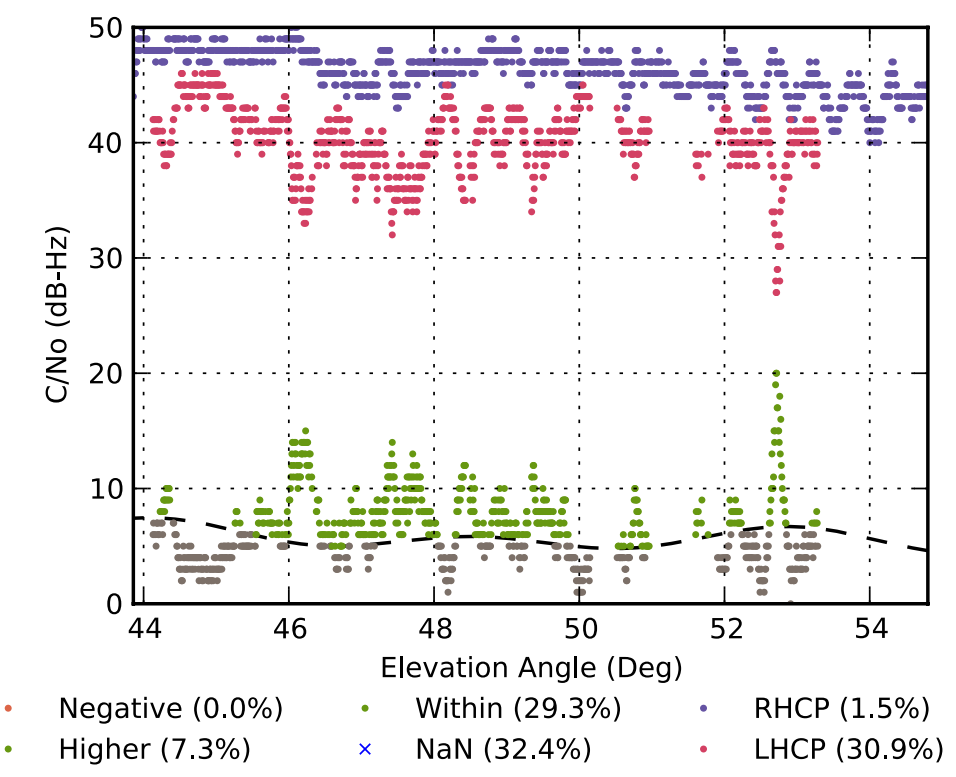

Fig.13 One identified severe multipath (G20) at T4

As shown in the figure, the identified signal is from a medium-elevation satellite. The results show that $31 \%$ of the overall reception period is contaminated with multipath signals worse than the Regent's Park environment, whereas $29.3 \%$ has minimal multipath contamination, similar to the Regent's Park environment. It can also be noted that $32.4 \%$ of the reception cannot be compared against the thresholds, as simultaneous reception on both feeds was not available. However, it can be assumed that there is very little multipath interference in cases where no signal can be tracked from the LHCP antenna feed.

The results shown in Fig.13 can also be verified by examining the physical surroundings of the T4 reception point. As demonstrated in the left panel of Fig.14, the T4 point is located on a street with a roughly east-west orientation. The satellite ground track of PRN 20 (Fig.14, right panel) suggests that the satellite should be rising from the east and moving towards the west. From the view of the receiver, the satellite should be located at roughly the along street direction moving overhead (as shown in centre panel of Fig.14). Because the satellite has a medium elevation, there would be a fair chance for the signal to be reflected. Therefore, a mixture of good and contaminated reception would be expected as demonstrated in Fig.13.
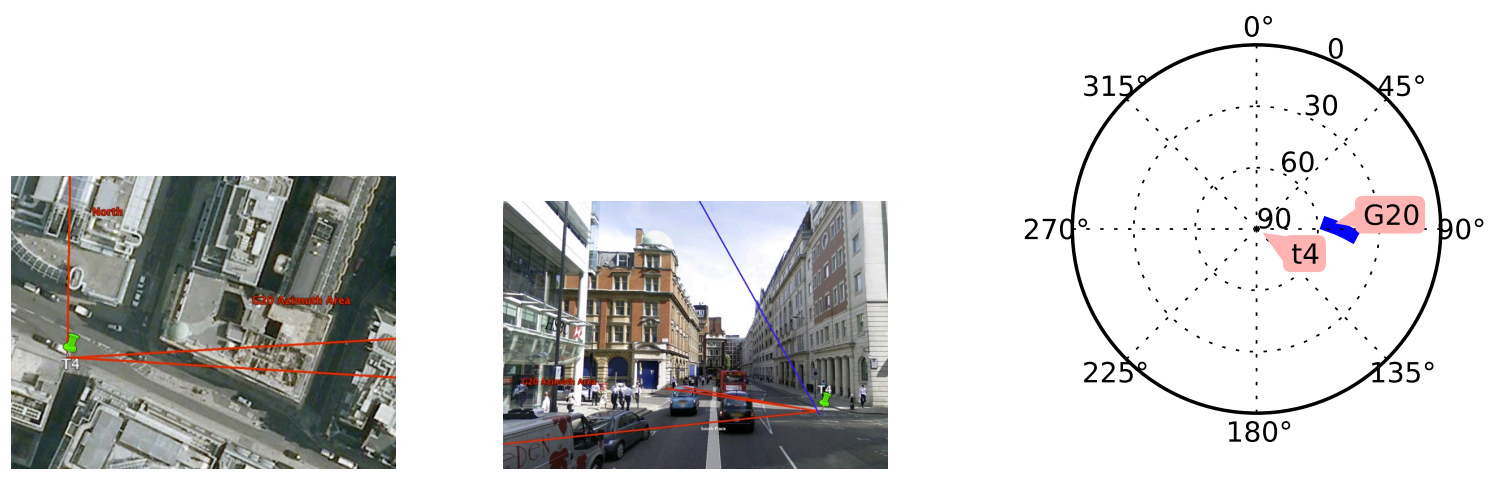

Fig.14 T4 site surroundings and G20 ground track

Fig. 15 shows an example of high-elevation signal reception. The signal was from PRN 13 at point T9. As can be seen from the graph, the $C / N_{0}$ differences for a major part $(64.5 \%)$ of the reception epoch lie above the lower $95 \%$ bond threshold. 


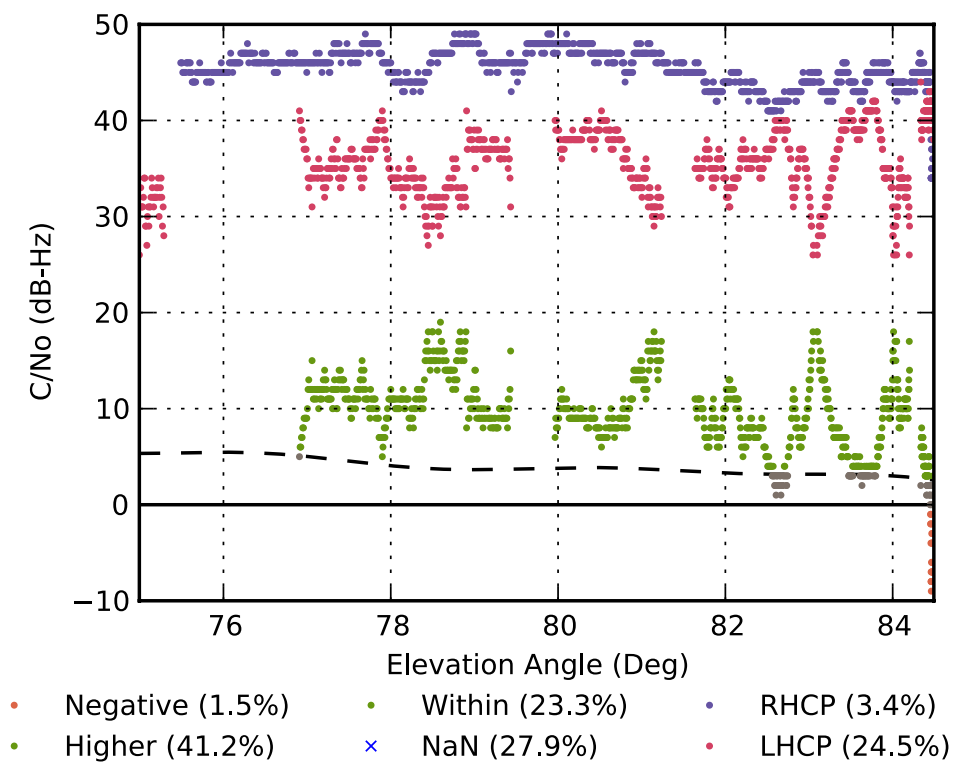

Fig.15 An example of high-elevation signal reception (G13) at T9
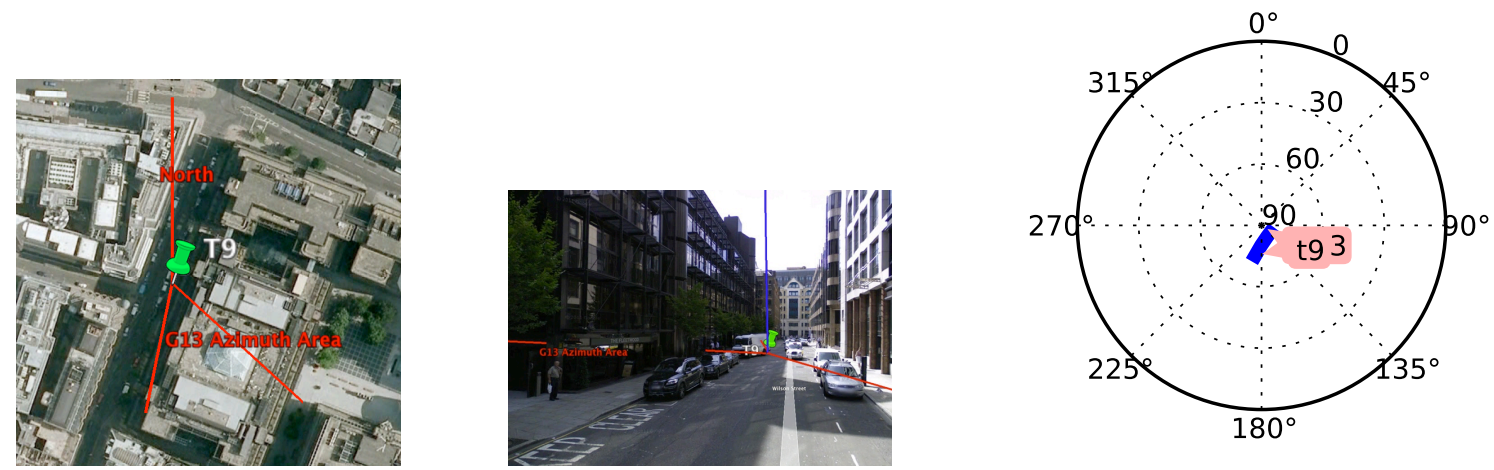

Fig.16 T9 site surroundings and G13 ground track

The physical surrounding of point T9 and the satellite skyplot are shown in Fig.16 following the same convention as in Fig.14. The satellite is moving almost directly overhead of the receiver with a high elevation as shown by the blue direct line-of-sight in centre panel of Fig. 16.

In summary, two signals (PRN 24 and 32) received at the point T3 were identified as suffering from severe NLOS reception. All other signals were classified as a mixture of low to medium or severe multipath-contaminated, with occasional period of NLOS reception.

\section{Impact On Position Accuracy}

Position solutions have been calculated for the point T3 both using all available measurements and excluding the identified NLOS signals. The single point solution is processed using a weighted least squares based algorithm. The weighting scheme is based on the satellite elevation. A single position solution is calculated using measurements made during the occupation period at point $\mathrm{T} 3$ at each epoch and is compared against the presurveyed coordinate.

A comparison of the mean positioning errors is shown in Fig.17. Positioning errors of East, North, Height and Horizontal directions are respectively plotted in blue, red, green and 
purple lines. Mean positioning errors of each direction are listed in the legend in meters. As can be seen from the top graph in Fig. 17, the positioning solutions using NLOS signals are erroneous. After excluding the NLOS signals (Fig.17, bottom graph), the positioning errors improved significantly, especially in the horizontal directions. However, the positioning accuracy is still worse than the standard performance of single frequency code-only solutions in an open-field environment. This is due to the extensive multipath contamination exhibited on many of remaining signals as demonstrated by the example in Fig.13 and 14.
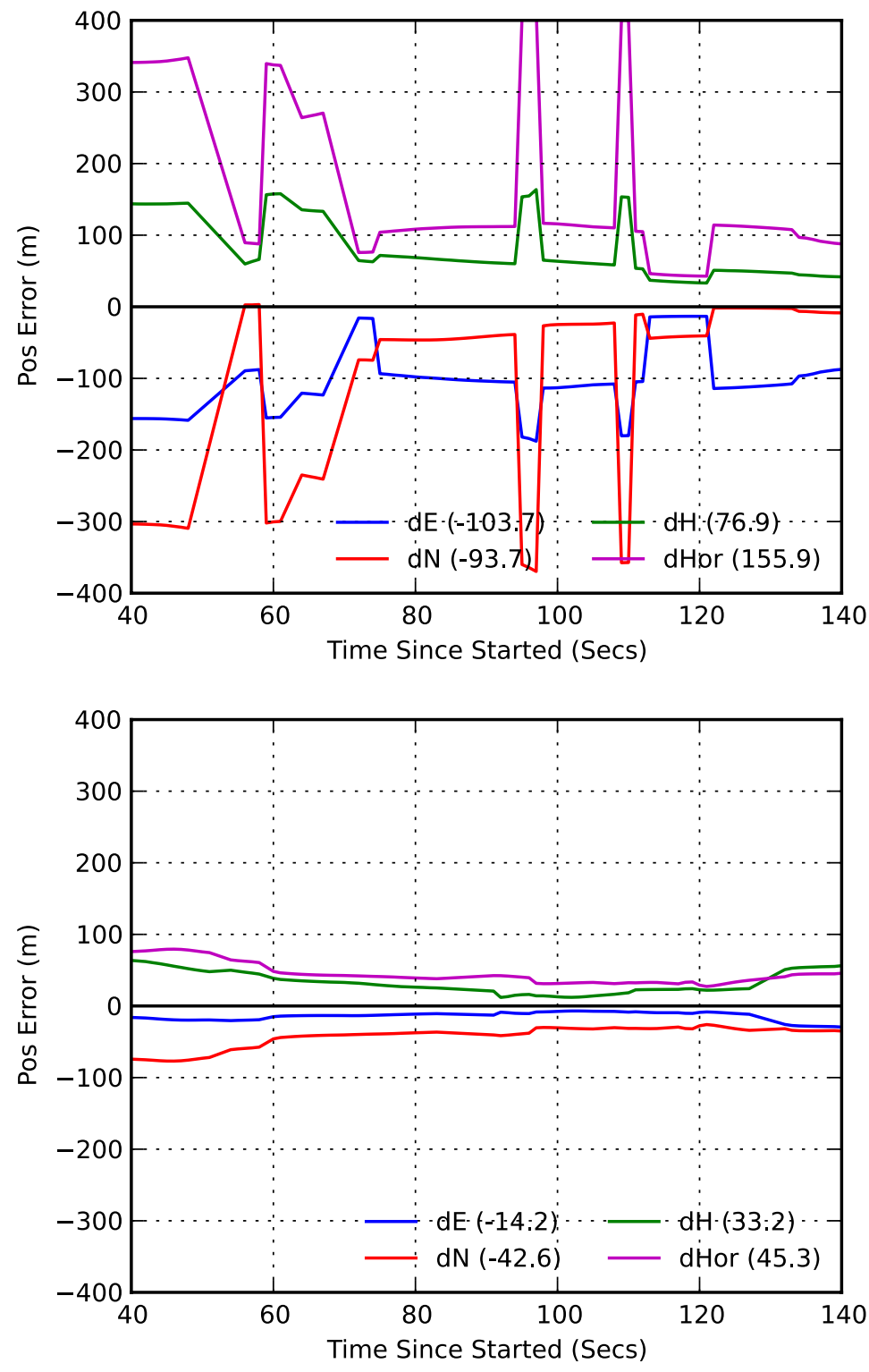

Fig.17 Position errors comparison with and without the identified NLOS signals at point T3

\section{Conclusions and Future Work}

The capability of NLOS signal detection using a dual-polarisation antenna has been clearly demonstrated. The $C / N_{0}$-based thresholds derived from the dual-polarisation data collected in a low multipath environments were used for the detection in an urban area. As NLOS reception tends to occur most frequently in urban positioning applications, especially for lowand medium-elevation signals, dense urban area data was used in the testing process. Two 
signals with NLOS-only reception were successfully identified among all received signals, and occasional NLOS reception was observed on other signals. The exclusion of the NLOS signals from the final positioning solution led to a significantly reduced error.

Currently, the application of the technique to multipath detection is limited by the requirement for an azimuth-dependent calibration process and knowledge of antenna orientation. A full azimuth- and elevation-dependent calibration can be performed in an Anechoic chamber using a GNSS signal simulator transmitting via antennas in the chamber. This would be expensive on an individual basis but is practical where measurements of one antenna may be applied to others of the same design. A variety of sensors may be used to determine the antenna orientation (Groves 2008).

The experimental results also demonstrated that severely degraded reception can affect a large portion of the signals received, leading to an erroneous position solutions when using only pseudoranges for positioning.

There are many topics that would benefit from further research. The current NLOS and severe multipath detection technique should be tested with a multi-constellation GNSS receiver. This will enable a greater proportion of the signals to be excluded from the positioning solution, with the potential to significantly improve accuracy. Testing should also be conducted using RTK carrier-phase positioning and while making use of an Extended Kalman Filter based positioning algorithm.

The response to doubly reflected signals should be investigated. If these revert to RHCP on the second reflection, the dual-polarisation technique will not detect them and additional NLOS/multipath detection techniques such as consistency checking (Jiang 2011) will be required. However, doubly reflected signals with mixed-polarisation should still be detectable using the dual-polarisation method.

Improvements to the dual-polarisation antenna itself should be investigated. A reduction in the variation of the LHCP gain with azimuth would enable greater multipath detection sensitivity without the need to orient the antenna. It might also enable the use of the dualpolarisation antenna to correct multipath as well as detect it as proposed in Yang (2005) and Groves (2010). Finally, miniaturisation of the antenna technology, if possible, would enable the technique to be applied in consumer devices, such as smart phones.

\section{Acknowledgements}

This work is part of the Innovative Navigation using new GNSS Signals with Hybridised Technologies (INSIGHT) program. INSIGHT (www.insight-gnss.org) is a collaborative research project funded by the UK's Engineering and Physical Sciences Research Council (EPSRC) and undertaken by Imperial College London, University College London, the University of Nottingham, the University of Westminster and 8 industrial partners.

The authors would like to thank Dr Yacine Adane from University of Westminster for designing the amplifier to work with the antenna, QinetiQ for lending the choke ring used in this research and the following people who assisted with the data collection: Mr Chris Atkins, Mr Lei Wang, and Ms Naomi Chian-yuan Li.

The authors would also like to thank Antom for providing the antenna test data and granting the permission for publishing the data. 


\section{Reference}

Brown, A., and Gerein, N. (2001) Test Results from a Digital P(Y) Code Beamsteering Receiver for Multipath Minimization. Proc. ION 57 ${ }^{\text {th }}$ Annual Meeting, Albuquerque, NM, $p$. 872-878.

Braasch, M. S. (1996) Multipath Effects. In: Global Positioning System: Theory and Applications Volume I. Washington, DC: AIAA, pp. 547-568.

Brenneman, M., Morton, J., Yang, C. and van Graas, F. (2011) Mitigation of GPS Multipath Using Polarization and Spatial Diversities. Proc. ION GNSS, Fort Worth, TX, pp. 1221-1229.

Groves, P. D. (2008) Principles of GNSS, Inertial and Multisensor Integrated Navigation Systems. Artech House.

Groves, P. D., Z. Jiang, et al. (2010) Novel Multipath Mitigation Methods using a Dualpolarization Antenna. Proc. ION GNSS, Portland, OR, pp. 140-151.

Hofmann-Wellenhof, B., Lichtenegger, H. and Wasle, E. (2008) GNSS--global navigation satellite systems: GPS, GLONASS, Galileo, and more. Springer Verlag Wien.

Izadpanah, A., O'Driscoll C. and G. Lachapelle (2008) GPS Multipath Parameterization using the Extended Kalman Filter and a Dual LHCP/RHCP Antenna. Proc. ION GNSS, Savannah, GA, pp. 689-697.

Jiang, Z., et al. (2011) Multi-Constellation GNSS Multipath Mitigation Using Consistency Checking. Proc. ION GNSS, Portland, OR, pp. 3889-3902.

Kaplan, E.D. and Hegarty, C.J. (2006) Understanding GPS: principles and applications. Artech House.

Manandhar, D., Shibasaki, R. and Normark P. (2004) GPS Signal Analysis using LHCP/RHCP Antenna and Software GPS Receiver. Proc. ION GNSS, Long Beach, CA, pp. 2480-2498.

Lau, L., and P. Cross (2007) Investigations into Phase Multipath Mitigation Techniques for High Precision Positioning in Difficult Environments. Journal of Navigation, Vol. 60, No. 1, pp. 95-105.

Van Nee, R. D. J. (1992) GPS Multipath and Satellite Interference. Proc. ION $48^{\text {th }}$ Annual Meeting, Washington, DC, pp. 167-177.

Wang, L., Groves, P.D., and Ziebart, M. K. (2012) Multi-Constellation GNSS Performance Evaluation for Urban Canyons Using Large Virtual Reality City Models. Journal of Navigation, Vol. 65, No. 3.

Yang, C., and A. Porter (2005) GPS Multipath Estimation and Mitigation Via Polarization Sensing Diversity: Parallel Iterative Cross Cancellation. Proc. ION GNSS, Long Beach, CA, pp. 2707-2719. 


\section{Author Biographies}

Dr Ziyi Jiang is a research fellow at University College London (UCL), currently specialising in multipath mitigation research in the Space Geodesy and Navigation Laboratory (SGNL). He completed his $\mathrm{PhD}$ at UCL on digital route model aided integrated satellite navigation and low-cost inertial sensors for high-performance positioning on the railways.

Dr Paul Groves is a Lecturer (academic faculty member) at UCL, where he leads a program of research into robust positioning and navigation. He is interested in all aspects of navigation and positioning, including multi-sensor integrated navigation, improving GNSS performance under challenging reception conditions, and novel positioning techniques. 